\title{
A NOTE ON EUCLIDEAN ORDER TYPES
}

\author{
PETE L. CLARK
}

\begin{abstract}
Euclidean functions with values in an arbitrary well-ordered set were first considered in a 1949 work of Motzkin and studied in more detail in work of Fletcher, Samuel and Nagata in the 1970's and 1980's. Here these results are revisited, simplified, and extended. The two main themes are (i) consideration of Ord-valued functions on an Artinian poset and (ii) use of ordinal arithmetic, including the Hessenberg-Brookfield ordinal sum. In particular, to any Euclidean ring we associate an ordinal invariant, its Euclidean order type, and we initiate a study of this invariant. The main new result gives upper and lower bounds on the Euclidean order type of a finite product of Euclidean rings in terms of the Euclidean order types of the factor rings.
\end{abstract}

Throughout, "a ring" means a commutative ring with multiplicative identity. We denote by $R^{\bullet}$ the set $R \backslash\{0\}$ and by $R^{\times}$the group of units of $R$. We denote by $\mathbb{N}$ the natural numbers, including 0 . When we are thinking of $\mathbb{N}$ as the least infinite ordinal, we denote it by $\omega$. If $S$ is any class equipped with a "zero element" - here, either the least element of an ordered class or the identity element of a monoid then we put $S^{\bullet}=S \backslash\{0\}$.

By an ordered set $X$ we mean a pair $(X, \leq)$ with $X$ a set and $\leq$ a reflexive, antisymmetric, transitive relation on $X$ (i.e., what is often called a partial ordering).

We will encounter some ordinal arithmetic, and it is important to remember that the "ordinary" sum and product of transfinite ordinal numbers need not be commutative. The literature seems to agree that for $\alpha, \beta \in$ Ord, $\alpha+\beta$ should be the order type of a copy of $\beta$ placed above a copy of $\alpha$, so that $\omega+1>\omega$, $1+\omega=\omega$. However, both conventions on $\alpha \beta$ seem to be in use. We take the one in which $2 \omega=\omega+\omega$, not the one in which $2 \omega=2+2+\ldots=\omega$.

\section{Ordered Classes, Isotone Maps, and Length Functions}

\subsection{Isotone Maps and Artinian Ordered Classes.}

For a set $X$, let $\operatorname{Ord}^{X}$ denote the class of all maps $f: X \rightarrow$ Ord, ordered by $f \leq g \Longleftrightarrow \forall x \in X, f(x) \leq g(x)$ 皿 Note that every nonempty subclass $\mathcal{C}=\left\{f_{c}\right\}$ has an infimum in $\operatorname{Ord}^{X}$ : that is, there is a largest element $f \in \operatorname{Ord}^{X}$ with the property that $f \leq f_{c}$ for all $f_{c} \in \mathcal{C}$. Indeed, we may take $f_{\mathcal{C}}(x)=\min _{c} f_{c}(x)$.

An ordered class $\mathcal{C}$ is downward small if for all $x \in \mathcal{C},\{y \in X \mid y \leq x\}$ is a set. For any set $X, \operatorname{Ord}^{X}$ is downward small.

Let $X$ and $Y$ be ordered classes. We denote by $X \times Y$ the Cartesian product

Thanks to David Krumm and Robert Varley for providing the inspiration for this work.

(C) Pete L. Clark, 2012.

${ }^{1}$ We hasten to reassure the reader that this is the limit of our set-theoretic ambitiousness: we will never consider the collection of all maps between two proper classes! 
endowed with the ordering $\left(x_{1}, y_{1}\right) \leq\left(x_{2}, y_{2}\right) \Longleftrightarrow x_{1} \leq x_{2}$ and $y_{1} \leq y_{2}$. A map $f: X \rightarrow Y$ is weakly isotone (resp. isotone) if $x_{1} \leq x_{2} \in X \Longrightarrow f\left(x_{1}\right) \leq f\left(x_{2}\right)$ (resp. $x_{1}<x_{2} \Longrightarrow f\left(x_{1}\right)<f\left(x_{2}\right)$ ). The composite of (weakly) isotone maps is (weakly) isotone.

An ordered class $X$ is Noetherian (resp. Artinian) if there is no isotone map $f: \mathbb{Z}^{+} \rightarrow X$ (resp. $f: \mathbb{Z}^{-} \rightarrow X$ ). Thus a well-ordered class is precisely a linearly ordered Artinian class.

For an ordered set $X$, we define $\operatorname{Iso}(X) \subset \operatorname{Ord}^{X}$ to be the subclass of isotone maps, with the induced partial ordering.

Lemma 1. Let $X$ be an ordered set. Then every nonempty subclass of $\operatorname{Iso}(X)$ has an infimum in $\operatorname{Iso}(X)$.

Proof. Let $\mathcal{C}=\left\{f_{c}\right\}$ be a nonempty subclass of Iso $(X)$, and let $f$ be the infimum in $\operatorname{Ord}^{X}$; it suffices to show that $f$ is isotone. If $x<y$ in $X$, then $f_{i}(x)<f_{i}(y)$ for all $i \in I$, so $f(x)=\min _{i \in I} f_{i}(x)<\min _{i \in I}\left(f_{i}(x)+1\right) \leq \min _{i \in I} f_{i}(y)=f(y)$.

Theorem 2. For a downward small ordered class $X$, TFAE:

(i) There is an isotone map $f: X \rightarrow$ Ord.

(ii) There is an Artinian ordered set $Y$ and an isotone map $f: X \rightarrow Y$.

(iii) $X$ is Artinian.

Proof. (i) $\Longrightarrow$ (ii): If $f: X \rightarrow \operatorname{Ord}(X)$ is an isotone map, then $f: X \rightarrow f(X)$ is an isotone map with codomain an Artinian ordered set.

(ii) $\Longrightarrow$ (iii): Suppose not: then there is an isotone map $\iota: \mathbb{Z}^{-} \rightarrow X$ is an isotone map. But then $f \circ \iota: \mathbb{Z}^{-} \rightarrow Y$ is isotone, so $Y$ is not Artinian.

(iii) $\Longrightarrow$ (i) [Na85, Prop. 4]: We will construct $\lambda_{X} \in \operatorname{Iso}(X)$ by a transfinite process. For $\alpha \in$ Ord, at the $\alpha$ th stage we assign some subset $X_{\alpha} \subset X$ the value $\alpha$. Let $X_{0}$ be the set of minimal elements of $X$. Having defined $X_{\beta}$ for all $\beta<\alpha$, we assign the value $\alpha$ to all $x \in X \backslash X_{\beta}$ such that there is $Y_{x} \subset X_{\beta}$ with $x=\sup Y_{x}$. Then, for arbitrary $X$, taking $X^{\prime}=\bigcup_{\alpha} X_{\alpha}$, this defines $\lambda_{X} \in \operatorname{Iso}\left(X^{\prime}\right)$. We claim that since $X$ is Artinian and downward small, $X^{\prime}=X$ : if not, let $x$ be a minimal element of $X \backslash X^{\prime}$. Then $\lambda_{X}$ is defined on $D^{\circ}(x)=\{y \in X \mid y<x\}$ and $x=\sup D^{\circ}(x)$, so if $\alpha=\sup \lambda_{X}\left(D^{\circ}(x)\right)$, then $\lambda_{X}(x)=\alpha$ (if $\alpha \notin \lambda_{X}\left(D^{\circ}(x)\right.$ ) or $\lambda_{X}(x)=\alpha+1$ (if $\alpha \in D^{\circ}(x)$ ).

\subsection{Length Functions.}

By Theorem 2 and Lemma1, for any Artininan ordered set $X$, Iso $(X)$ has a bottom element. Indeed the map $\lambda_{X}: X \rightarrow$ Ord constructed in the proof of Theorem 2 is the bottom element of Iso $(X)$. Following [Br02, we call $\lambda_{X}$ the length function.

If $X$ has a top element $T$, we define the length of $\mathbf{X}$ to be len $(X)=\lambda_{X}(T)$.

Example 1.1: For $\alpha \in$ Ord and $x \leq \alpha, \lambda_{\alpha+1}(x)=x$, so len $(\alpha+1)=\lambda_{\alpha+1}(\alpha)=\alpha$.

Example 1.2: For $m, n \in \mathbb{Z}^{+}$, let $X_{1}=\{0, \ldots, m\}$ and $X_{2}=\{0, \ldots, n\}$, and let $X=X_{1} \times X_{2}$. Then for all $(i, j) \in X, \lambda_{X}(i, j)=i+j$ and $\operatorname{len}(X)=m+n$. 
For $\alpha, \beta \in$ Ord, we define the Brookfield sum

$$
\alpha \oplus_{B} \beta:=\operatorname{len}((\alpha+1) \times(\beta+1)) .
$$

We recall the following, a version of the Cantor normal form: for any $\alpha, \beta \in$ Ord there are $\gamma_{1}, \ldots, \gamma_{r} \in$ Ord, $r \in \mathbb{Z}^{+}$and $m_{1}, \ldots, m_{r}, n_{1}, \ldots, n_{r} \in \mathbb{N}$ with

$$
\alpha=m_{1} \omega^{\gamma_{1}}+\ldots+m_{r} \omega^{\gamma_{r}}, \beta=n_{1} \omega^{\gamma_{1}}+\ldots+n_{r} \omega^{\gamma_{r}}
$$

This representation of the pair $(\alpha, \beta)$ is unique if we $\operatorname{require} \max \left(m_{i}, n_{i}\right)>0$ for all $i$. We may then define the Hessenberg sum

$$
\alpha \oplus_{H} \beta=\left(m_{1}+n_{1}\right) \omega^{\gamma_{1}}+\ldots+\left(m_{r}+n_{r}\right) \omega^{\gamma_{r}} .
$$

Theorem 3. For all $\alpha, \beta \in$ Ord, $\alpha \oplus_{B} \beta=\alpha \oplus_{H} \beta$.

Proof. See [Br02, Thm. 2.12].

In view of Theorem 3 we write $\alpha \oplus \beta$ for $\alpha \oplus_{B} \beta=\alpha \oplus_{H} \beta$ and speak of the Hessenberg-Brookfield sum. This operation is well-known to the initiates of ordinal arithmetic, who call it the "natural sum". The next result collects facts about $\alpha \oplus \beta$ for our later use.

Proposition 4. Let $\alpha, \beta \in$ Ord.

a) If $\beta<\omega$, then $\alpha+\beta=\alpha \oplus \beta$.

b) In general we have

$$
\max (\alpha+\beta, \beta+\alpha) \leq \alpha \oplus \beta \leq \alpha \beta+\beta \alpha .
$$

Proof. Left to the reader.

\section{Euclidean Functions}

\subsection{Basic Definitions.}

A Euclidean function is a function $\varphi: R^{\bullet} \rightarrow$ Ord such that for all $a \in R$, $b \in R^{\bullet}$, there are $q, r \in R$ with $a=q b+r$ and $(r=0$ or $\varphi(r)<\varphi(b))$. A ring is Euclidean if it admits a Euclidean function.

Example 2.1: Let $R=\mathbb{Z}$. Then $n \mapsto|n|$ is a Euclidean function.

Example 2.2: Let $k$ be a field and let $R=k[t]$. Then $\varphi: R^{\bullet} \rightarrow \mathbb{Z}^{+}$given by $P \mapsto 1+\operatorname{deg} P$ is a Euclidean function, as is $P \mapsto 2^{\operatorname{deg} P}$.

Example 2.3: Sa71, Prop. 5] Let $R$ be a semilocal PID with nonassociate prime elements $\pi_{1}, \ldots, \pi_{n}$. We may write $x \in R^{\times}$as $u \pi_{1}^{a_{1}} \cdots \pi_{n}^{a_{n}}$ for $a_{1}, \ldots, a_{n} \in \mathbb{N}$, and then $x \mapsto a_{1}+\ldots+a_{n}$ is a Euclidean function.

Proposition 5. a) Let $\varphi: R^{\bullet} \rightarrow$ Ord be a Euclidean function. For every nonzero ideal $I$ of $R$, let $x \in I^{\bullet}$ be such that $\varphi(x) \leq \varphi(y)$ for all $y \in I^{\bullet}$. Then $I=\langle x\rangle$.

b) In particular, a Euclidean ring is principal.

Proof. Left to the reader; or see [Sa71, Prop. 3].

Extension at zero: It will be convenient to define our Euclidean functions at the zero element of $R$. There are several reasonable ways to do this. Although the initially appealing one is to take $\varphi(0)=0$ and require $\varphi$ to take nonzero values on 
$R^{\bullet}$, in the long run it turns out to be useful to take a quite different convention: we allow Euclidean functions to take the value zero at nonzero arguments - so that, in particular, the bottom Euclidean function $\varphi_{R}$ will take the value 0 precisely at the units - and we define $\varphi(0)=\sup _{x \in R} \bullet \varphi(x)+1$. This is actually not so strange: after all, 0 is the top element of $R$ with respect to the divisibility quasi-ordering.

\subsection{Structure Theory of Principal Rings.}

Theorem 6. a) If $R=\prod_{i=1}^{r} R_{i}$, then $R$ is principal iff $R_{i}$ is principal for all $i$. b) For every principal ring $R$ there is $r \in \mathbb{N}$, a finite set of principal ideal domains $R_{1}, \ldots, R_{n}$ and a principal Artinian ring $A$ such that $R \cong \prod_{i=1}^{n} R_{i} \times A$. The $R_{i}$ 's are uniquely determined by $R$ up to isomorphism (and reordering), and $A$ is uniquely determined by $R$ up to isomorphism: we call $A$ the Artinian part of $R$. c) A ring is Artinian principal iff it is isomorphic to a finite product of local Artinian principal rings.

Proof. See [ZS, p. 245].

\subsection{Generalized Euclidean Functions.}

A generalized Euclidean function is a function $\varphi$ from $R^{\bullet}$ to an Artinian ordered class $X$ such that for all $x \in R, y \in R^{\bullet}$, there are $q, r \in R$ with $a=q x+r$ such that either $r=0$ or $\varphi(r)<\varphi(b)$.

As several authors have observed over the years, nothing in the theory of Euclidean functions is lost by entertaining Euclidean functions with values in any Artinian ordered class. In particular, the proof of Proposition 5 carries over easily to show that a ring admitting a generalized Euclidean function is principal. But there is a better way to see this:

Lemma 7. Let $X, Y$ be Artinian ordered classes, $\varphi: R \rightarrow X$ a generalized Euclidean function and $f: X \rightarrow Y$ an isotone map. Then $f \circ \varphi: R \rightarrow Y$ is a generalized Euclidean function.

Proof. Left to the reader.

Corollary 8. ([Sa71, Prop. 11], Na85, Prop. 4]) A ring which admits a generalized Euclidean function is Euclidean.

Proof. If $\varphi: R \rightarrow X$ is generalized Euclidean, $\lambda_{X} \circ \varphi: R \rightarrow$ Ord is Euclidean.

Proposition 8 may suggest that there is nothing to gain in considering Euclidean functions with values in a non-well-ordered sets. But this is not the case!

Lemma 9. ([Na85, Thm. 2]) Let $R_{1}, R_{2}$ be commutative rings, $X_{1}, X_{2}$ be Artinian ordered classes, and $\varphi_{1}: R_{1} \rightarrow X_{1}, \varphi_{2}: R_{2} \rightarrow X_{2}$ be generalized Euclidean functions. Then $\varphi_{1} \times \varphi_{2}: R_{1} \times R_{2} \rightarrow X_{1} \times X_{2}$ is a generalized Euclidean function.

Proof. Let $x=\left(x_{1}, x_{2}\right) \in R, y=\left(y_{1}, y_{2}\right) \in R^{\bullet}$. We may assume $y \nmid x$ and thus $x \in R^{\bullet}$. Since $\varphi_{1}$ and $\varphi_{2}$ are Euclidean, for $i=1,2$ there are $q_{i}, r_{i} \in R_{i}$ with $x_{i}=q_{i} y_{i}+r_{i}$ and $\left(r_{i}=0\right.$ or $\left.\varphi_{i}\left(r_{i}\right)<\varphi_{i}\left(y_{i}\right)\right)$. Since $y \nmid x, r \neq 0$. Now:

Case 1: Suppose that any one of the following occurs:

(i) $r_{1} \neq 0, r_{2} \neq 0$. 
(ii) $r_{1}=y_{1}=0$, and thus $r_{2}, y_{2} \neq 0$.

(iii) $r_{2}=y_{2}=0$, and thus $r_{1}, y_{1} \neq 0$.

Put $q=\left(q_{1}, q_{2}\right)$ and $r=\left(r_{1}, r_{2}\right)$, so $x=q y+r$ and

$$
\varphi(r)=\left(\varphi\left(r_{1}\right), \varphi\left(r_{2}\right)\right)<\left(\varphi\left(y_{1}\right), \varphi\left(y_{2}\right)\right)=\varphi(y) .
$$

Case 2: Suppose $r_{1}=0, y_{1} \neq 0$, and thus $r_{2} \neq 0$. Then take $q=\left(q_{1}-1, q_{2}\right)$ and $r=\left(y_{1}, r_{2}\right)$, so $x=q y+r$ and

$$
\varphi(r)=\left(\varphi\left(y_{1}\right), \varphi\left(r_{2}\right)\right)<\left(\varphi\left(y_{1}\right), \varphi\left(y_{2}\right)\right)=\varphi(y) .
$$

Similarly if $r_{2}=0, y_{2} \neq 0$.

Case 3: Suppose $r_{2}=0, y_{2} \neq 0$, and thus $r_{1} \neq 0$. Put $q=\left(q_{1}, q_{2}-1\right)$ and $r=\left(r_{1}, y_{2}\right)$, so $x=q y+r$ and

$$
\varphi(r)=\left(\varphi\left(r_{1}\right), \varphi\left(y_{2}\right)\right)<\left(\varphi\left(y_{1}\right), \varphi\left(y_{2}\right)\right)=\varphi(y) .
$$

\subsection{Isotone Euclidean Functions.}

For a ring $R$, the divisibility relation is a quasi-ordering - i.e., reflexive and transitive but not necessarily anti-symmetric. If $X$ and $Y$ are quasi-ordered sets, we can define an isotone map $f: X \rightarrow Y$ just as above: if $x_{1}<x_{2} \Longrightarrow f\left(x_{1}\right)<f\left(x_{2}\right)$.

Lemma 10. Let $X$ be a quasi-ordered set and $Y$ be an ordered set. Suppose that $f: X \rightarrow Y$ is an isotone map. Then $X$ is ordered.

Proof. For $x_{1}, x_{2} \in X, x_{1}<x_{2}, x_{2}<x_{1} \Longrightarrow f\left(x_{1}\right)<f\left(x_{2}\right), f\left(x_{2}\right)<f\left(x_{1}\right)$.

If $X$ is a quasi-ordered set, it has an ordered completion: i.e., an ordered set $\bar{X}$ and a weakly isotone map $X \rightarrow \bar{X}$ which is universal for weakly isotone maps from $X$ into an ordered set. Indeed, we simply take the quotient of $X$ under the equivalence relation $x_{1} \sim x_{2}$ if $x_{1} \leq x_{2}$ and $x_{2} \leq x_{1}$. If we do this for the divisibility relation on $R$, we get precisely the ordered set Prin $R$ of principal ideals of $R$. All this is to motivate the following definition.

A Euclidean function $\varphi: R \rightarrow$ Ord is weakly isotone (resp. isotone) if whenever $x$ divides $y, \varphi(x) \leq \varphi(y)$ (resp. whenever $x$ strictly divides $y, \varphi(x)<\varphi(y)$.

Example 2.4: Define $\varphi: \mathbb{Z}^{\bullet} \rightarrow$ Ord $^{\bullet}$ by $1 \mapsto 2$ and $n \mapsto|n|$ else. Then $\varphi$ is Euclidean. But $(1)=(-1)$ and $\varphi(1) \neq \varphi(-1)$, so $\varphi$ is not weakly isotone.

Theorem 11. Let $\varphi: R^{\bullet} \rightarrow$ Ord be a Euclidean function. Then the set of isotone Euclidean functions $\psi \leq \varphi$ has a maximal element

$$
\underline{\varphi}: x \in R^{\bullet} \mapsto \min _{y \in(x)} \varphi(y) .
$$

Proof. Step 1: We show $\underline{\varphi}$ is a Euclidean function: let $a \in R, b \in R^{\bullet}$. Then there exists $c \in R$ such that $b \bar{c} \neq 0$ and $\underline{\varphi}(b)=\varphi(b c)$. Since $\varphi$ is Euclidean, there are $q, r \in R$ with $a=q b c+r$ and either $\bar{r}=0-$ so $b \mid a-$ or $\underline{\varphi}(r) \leq \varphi(r)<\varphi(b c)=\underline{\varphi}(b)$. Step 2: We show $\underline{\varphi}$ is isotone: If $a c \neq 0$, then $\underline{\varphi(a)}=\min _{y} \mid a y \neq 0 \quad \varphi(a \bar{y}) \leq$ $\min _{c y \mid a c y \neq 0} \varphi(a c y)=\underline{\varphi}(a c)$. Conversely, suppose $\underline{\varphi}(a c)=\underline{\varphi}(a)$, and write $a=$ $q a c+r$ with $r=0$ or $\underline{\varphi}(r)<\underline{\varphi}(a c)=\underline{\varphi}(a)$. But if $\bar{r} \neq 0$, then by what we have just shown, $\underline{\varphi}(r)=\underline{\varphi}(a(1-q c)) \geq \underline{\varphi}(a)$, a contradiction. So $r=0$ and $(a)=(a c)$. Step 3: By construction $\underline{\varphi} \leq \varphi$. Moreover, if $\psi \leq \varphi$ is an isotone Euclidean function, then for all $a, c \in R$ with $a c \neq 0, \psi(a) \leq \psi(a c) \leq \varphi(a c)$, so $\psi(a) \leq \underline{\varphi}(a)$. 
Corollary 12. A Euclidean function is weakly isotone iff it is isotone.

Proof. Of course any isotone function is weakly isotone. Conversely, let $\varphi: R^{\bullet} \rightarrow$ Ord be a weakly isotone Euclidean function, let $a, c \in R$ with $a c \neq 0$, and suppose $\varphi(a c)=\varphi(c)$. Write $a=q a c+r$ with $r=0$ or $\varphi(r)<\varphi(a c)=\varphi(a)$. If $r \neq 0$, then $\varphi(r)=\varphi(a(1-q c)) \geq \varphi(a)$, contradiction. So $r=0$ and $(a)=(a c)$.

\subsection{The Bottom Euclidean Function and the Euclidean Order Type.}

For any commutative ring $R$, let $\operatorname{Euc}(R) \subset \operatorname{Ord}^{R}$ be the subclass of Euclidean functions $\varphi: R \rightarrow$ Ord, with the induced partial ordering.

Lemma 13. Let $R$ be a commutative ring. Then every nonempty subclass of $\operatorname{Euc}(R)$ has an infimum in $\operatorname{Euc}(R)$.

Proof. Let $\mathcal{C}=\left\{\varphi_{c}\right\}$ be a nonempty subclass of $\operatorname{Euc}(R)$, and let $\varphi$ be the infimum in $\operatorname{Ord}^{R}$; it suffices to show that $\varphi$ is Euclidean. Let $a, b \in R$ with $b \nmid a$. Choose $i \in I$ such that $\varphi(b)=\varphi_{i}(b)$. Since $\varphi_{i}$ is Euclidean, there are $q, r \in R$ such that $\varphi_{i}(r)<\varphi_{i}(b)$, and then $\varphi(r) \leq \varphi_{i}(r)<\varphi_{i}(b)=\varphi(b)$.

Theorem 14. Let $R$ be a Euclidean ring.

a) The class $\operatorname{Euc}(R)$ of all Euclidean functions on $R$ has a bottom element $\varphi_{R}$.

b) The bottom Euclidean function $\varphi_{R}$ is isotone.

c) The set $\varphi_{R}(R)$ is an ordinal.

Proof. a) This is immediate from Lemma 13.

b) Since $\varphi_{R}$ is the bottom Euclidean function, we must have $\varphi_{R}=\underline{\varphi_{R}}$.

c) It's enough to show $\varphi_{R}(R)$ is downward closed: we need to rule out the existence of $\alpha<\beta \in$ Ord such that $\varphi_{R}(R)$ contains $\beta$ but not $\alpha$. Let $\alpha^{\prime}$ be the least element of $\varphi_{R}(R)$ exceeding $\alpha$. If we redefine $\varphi_{R}$ to take the value $\alpha$ whenever $\varphi_{R}$ takes the value $\alpha^{\prime}$, we get a smaller Euclidean function than $\varphi_{R}$ : contradiction.

Proposition 15. If $R$ is Euclidean, the bottom Euclidean function $\varphi_{R}$ is isotone.

Proof. By Theorem [11, $\varphi_{R}$ is an isotone Euclidean function. But $\varphi_{R}$ is the bottom Euclidean function, so $\underline{\underline{\varphi_{R}}}=\varphi_{R}$.

For any Euclidean ring $R$ we define the order type $e(R)=\varphi_{R}(R) \in$ Ord. This ordinal invariant of $R$ is our main object of interest: given a Euclidean ring $R$ we would like to compute its order type $e(R)$; conversely we would like to know which ordinals arise as order types of Euclidean rings.

Example 2.5: Let $R=\mathbb{Z}$. The map $n \in \mathbb{Z} \mapsto \#$ of binary digits of $|n|$ is the bottom Euclidean function, so $e(\mathbb{Z})=\omega$.

Example 2.6: Let $R=k[t]$. The map $x \in k[t] \mapsto 1+\operatorname{deg} t$ is the bottom Euclidean function, so $e(k[t]))=\omega$.

Theorem 16. Suppose $R$ is Euclidean with $e(R) \leq \omega$. Then:

a) For all $x \in R^{\bullet}, R /(x)$ is Artinian.

b) $R$ is either a PID or an Artinian principal ring. 
Proof. a) If $R /(x)$ were not Artinian, there would be a sequence of elements $\left\{x_{n}\right\}_{n=0}^{\infty}$ in $R$ with $x_{0}=x$ and $\left(x_{n+1}\right) \subsetneq\left(x_{n}\right)$ for all $n \in \mathbb{N}$. Applying Corollary 12b) we get $\varphi(x)=\varphi\left(x_{0}\right)>\varphi\left(x_{1}\right)>\ldots>\varphi\left(x_{n}\right)>\ldots$, contradicting $\varphi(x) \in \omega$.

b) This follows immediately from Theorem 6 ,

Corollary 17. (Fletcher [Fl71])

a) If $A$ is an Artinian Euclidean ring, then $e(R)<\omega$.

b) A Euclidean ring $R$ with $e(R)=\omega$ is a domain.

Proof. a) The value of $\varphi_{A}$ at $x \in R$ depends only on the ideal $(x)$. But an Artinian principal ring has only finitely many ideals! So $e(R)<\omega$.

b) This follows immediately from Theorem 16b) and part a).

\subsection{The Localized Euclidean Function.}

Theorem 18. Let $R$ be a Euclidean domain, and let $S \subset R$ be a multiplicatively closed subset. Then the localization $S^{-1} R$ is Euclidean and $e\left(S^{-1} R\right) \leq e(R)$.

Proof. See [Mo49, §4] or Samuel [Sa71, Prop. 7].

\subsection{The Quotient Euclidean Function.}

Theorem 19. Let $\varphi: R \rightarrow$ Ord be a Euclidean function. Let $b \in R^{\bullet}$ be an ideal of $R$, and let $f: R \rightarrow R /(b)$ be the quotient map. For $x \in R /(b)$, let $\tilde{x} \in f^{-1}(x)$ be any element such that $\varphi(\tilde{x}) \leq \varphi(y)$ for all $y \in f^{-1}(x)$.

a) Then $\varphi^{\prime}: x \in(R /(b))^{\bullet} \mapsto \varphi(\tilde{x})$ is a Euclidean function.

b) For the bottom Euclidean function $\varphi_{R}$, we have

$$
\varphi_{R}^{\prime}(0)=\sup _{\bar{x} \neq \overline{0}} \varphi_{R}^{\prime}(\bar{x})+1=\varphi_{R}(b) .
$$

Proof. a) For $x \in R /(b), y \in(R /(b))^{\bullet}, \tilde{x} \in R, \tilde{y} \in R^{\bullet}$, so there are $q, r \in R$ with $\tilde{x}=q \tilde{y}+r$ and $\varphi(r)<\varphi(\tilde{y})$. Then $x=f(q) y+f(r)$ and hence

$$
\varphi^{\prime}(f(r)) \leq \varphi(r)<\varphi(\tilde{y})=\varphi^{\prime}(y) .
$$

b) The first equality in (2) is the definition of the extension to 0 of any Euclidean function. The second equality is a key - in fact, characteristic - property of the bottom Euclidean function which is proved in [Sa71, Prop. 10].

Corollary 20. If $R$ is Euclidean, so is every quotient ring $R^{\prime}$, and $e\left(R^{\prime}\right) \leq e(R)$.

\subsection{The Product Theorem.}

Lemma 21. (Ordinal Subtraction)

a) For $\alpha \leq \beta \in$ Ord, there is a unique $\gamma \in$ Ord such that $\alpha+\gamma=\beta$. We may therefore define

$$
-\alpha+\beta=\gamma
$$

b) Suppose we have ordinals $\alpha, \beta, \gamma$ such that $\gamma \leq \alpha<\beta$. Then $-\gamma+\alpha<-\gamma+\beta$.

Proof. a) Existence of $\gamma$ : If $\alpha=\beta$, then we take $\gamma=0$. Otherwise, $\alpha \subsetneq \beta$; let $x_{0}$ be the least element of $\beta \backslash \alpha$ and let $\gamma$ be the order type of $\left\{x \in \beta \mid x \geq x_{0}\right\}$.

Uniqueness of $\gamma$ : suppose we have two well-ordered sets $W_{1}$ and $W_{2}$ such that $\alpha+W_{1}$ is order-isomorphic to $\alpha+W_{2}$. Then the unique order-isomorphism between them induces an order-isomorphism from $W_{1}$ to $W_{2}$.

b) For if not, $-\gamma+\beta \leq-\gamma+\alpha$, and then $\beta=\gamma+(-\gamma+\beta) \leq \gamma+(-\gamma+\alpha)=\alpha$. 
Theorem 22. (Product Theorem) Let $R_{1}, \ldots, R_{n}$ be Euclidean rings.

a) The ring $\prod_{i=1}^{n} R_{i}$ is Euclidean iff $R_{i}$ is Euclidean for all $i$.

b) If the equivalent conditions of part a) hold, then

$$
e\left(R_{1}\right)+\ldots+e\left(R_{n}\right) \leq e\left(\prod_{i=1}^{n} R_{i}\right) \leq e\left(R_{1}\right) \oplus \ldots \oplus e\left(R_{n}\right) .
$$

Proof. Induction reduces us to the case $n=2$. Put $R=R_{1} \times R_{2}$.

a) This is immediate from Lemma 9 . Corollary 8 and Theorem 19

b) Let $b=(0,1)$, so $R /(b)=R_{1}$ and thus by Theorem 19b), we have $\varphi_{R}(b)=e\left(R_{1}\right)$. For $y \in R_{2}, b=(0,1) \mid(0, y)$, so by Proposition [15, $\varphi_{R}((0,1)) \leq \varphi_{R}((0, y))$. By Lemma 21 we may put

$$
\psi(y)=-\varphi_{R}((0,1))+\varphi_{R}((0, y)) .
$$

We ClAim $\psi: R_{2} \rightarrow$ Ord is a Euclidean function. Granting this for the moment, it then follows that $\psi \geq \varphi_{R_{2}}$, so

$$
e(R)=\varphi_{R}((0,0))=\varphi_{R}((0,1))+\psi(0) \geq e\left(R_{1}\right)+e\left(R_{2}\right) .
$$

PROOF OF ClAIM: Let $x \in R_{2}, y \in R_{2}^{\bullet}$; as usual, we may assume $y \nmid x$. Since $\varphi_{R}$ is Euclidean, there are $q=\left(q_{1}, q_{2}\right), r=\left(r_{1}, r_{2}\right) \in R$ such that $(0, x)=q(0, y)+r=$ $\left(r_{1}, q_{2} y+r_{2}\right)$ and either $r=0$ or $\varphi_{R}(r)<\varphi_{R}((0, y))$. Thus $r_{1}=0$ and $x=q_{2} y+r_{2}$. Since $y \nmid x$ we have $r_{2} \neq 0$, so $r \neq 0$ and thus $\varphi_{R}\left(\left(0, r_{2}\right)\right)<\varphi_{R}((0, y))$. By Lemma 21b) we may subtract $\varphi_{R}(0,1)$ - on the left! - from both sides to get

$$
\psi\left(r_{2}\right)=-\varphi_{R}((0, l))-\varphi_{R}\left(\left(0, r_{2}\right)\right)<-\varphi_{R}((0,1))-\varphi_{R}((0, y))=\psi(y) .
$$

For the second inequality of (3), let $\varphi_{1}: R_{1} \rightarrow e\left(R_{1}\right), \varphi_{2}: R_{2} \rightarrow e\left(R_{2}\right)$ be the bottom Euclidean functions on $R_{1}$ and $R_{2}$. By definition of the Hessenberg-Brookfield sum, we have an isotone map $\lambda:\left(e\left(R_{1}\right)+1\right) \times\left(e\left(R_{2}\right)+1\right) \rightarrow e\left(R_{1}\right) \oplus e\left(R_{2}\right)$ and thus a Euclidean function $\lambda \circ\left(\varphi_{1} \times \varphi_{2}\right): R \rightarrow e\left(R_{1}\right) \oplus e\left(R_{2}\right)$.

Remark 2.7: The upper bound on the order type of the product ring in (3) is essentially due to Nagata. Moreover, in the proof of Proposition 6 of Sa71, Samuel gives the bound $e\left(R_{1} \times R_{2}\right) \leq e\left(R_{1}\right) \times e\left(R_{2}\right)+e\left(R_{2}\right) \times e\left(R_{1}\right)$. By Proposition $\left.4 \mathrm{~b}\right)$, Samuel's bound is not as good as Nagata's: there is a penalty to pay for employing the "usual" ordinal operations rather than the Hessenberg-Brookfield sum.

\subsection{Applications.}

Let $R$ be a nonzero local principal ring with maximal ideal $(\pi)$. Then every element $x \in R^{\bullet}$ may be written as $u \pi^{a}$ for $u \in R^{\times}$and a unique $a \in \mathbb{N}$. If $R$ is a domain, then we take the convention that $0=1 \cdot \pi^{\omega}$. Otherwise $R$ is Artinian and there is a least positive integer $a$ such that $0=1 \cdot \pi^{a}$; this $a$ is nothing else than $\ell(R)$, the length of $R$ as an $R$-module.

Theorem 23. a) (Samuel) Let $R$ be a local principal ring with maximal ideal generated by $\pi$. The map $\varphi: R \rightarrow$ Ord by $u \pi^{a} \mapsto a$ is a Euclidean function on $R$. b) In fact $\varphi=\varphi_{R}$ is the bottom Euclidean function, hence:

- If $R$ is a domain, $e(R)=\omega$.

- If $R$ is Artinian, then $e(R)=\ell(R)$. 
Proof. a) Let $x=u_{1} \pi^{a} \in R, y=u_{2} \pi^{b} \in R^{\bullet}$. If $b \leq a$ then $y \mid x$ and we may write $x=q y+0$. If $b>a$ we may write $x=0 \cdot y+x$, and then $\varphi(x)<\varphi(y)$.

b) Let $\varphi_{R}$ be the bottom Euclidean function on $R$, which is isotone by Proposition 15. Suppose first that $R$ is a domain. Then for all $a \in \omega$ we must have

$$
0=\varphi_{R}\left(\pi^{0}\right)<\varphi_{R}\left(\pi^{1}\right)<\ldots<\varphi_{R}\left(\pi^{a}\right)<\ldots
$$

and thus $\varphi \leq \varphi_{R}$, so $\varphi_{R}=\varphi$ and

$$
e(R)=\varphi(0)=\sup _{a \in \mathbb{N}} \varphi\left(\pi^{a}\right)+1=\omega .
$$

Similarly, if $R$ is Artinian then

$$
0=\varphi_{R}\left(\pi^{0}\right)<\varphi_{R}\left(\pi^{1}\right)<\ldots<\varphi_{R}\left(\pi^{\ell(R)}\right),
$$

and as above this forces $\varphi_{R}=\varphi$ and $e(R)=\varphi(0)=\ell(R)$.

Corollary 24. If $R$ is an Artinian principal ring, then $R$ is Euclidean and $e(R)=$ $\ell(R)$, the length of $R$ as an $R$-module.

Proof. This follows immediately from Theorem 23 and Theorem 22 .

Theorem 25. Let $R=\prod_{i=1}^{r} R_{i} \times A=R^{\prime} \times A$ be a Euclidean ring.

a) We have $e(R)=e\left(R^{\prime}\right)+\ell(A)$.

b) $e\left(R^{\prime}\right)$ is a limit ordinal (possibly zero).

c) $e\left(R^{\prime}\right) \geq r \omega$.

Proof. a) By Corollary 24, $A$ is Euclidean with $e(A)=\ell(A)$; then by Theorem 22,

$e\left(R^{\prime}\right)+\ell(A)=e\left(R^{\prime}\right)+e(A) \leq e(R) \leq e\left(R^{\prime}\right) \oplus e(A)=e\left(R^{\prime}\right)+e(A)=e\left(R^{\prime}\right)+\ell(A)$.

The remaining assertions hold trivially if $r=0$, so we assume $r \geq 1$.

b) Since $R^{\prime}$ is a product of domains, the set of nonzero ideals of $R$ has no minimal element, so $e\left(R^{\prime}\right)$ is a nonzero limit ordinal.

c) By part b) and Theorem 22, $r \omega \leq e\left(R_{1}\right)+\ldots+e\left(R_{r}\right) \leq e\left(R^{\prime}\right)$.

Corollary 26. (Fletcher) A Euclidean ring $R$ with $e(R)=\omega$ is a domain.

We say a Euclidean ring $R \cong \prod_{i=1}^{r} R_{i} \times A(R)$ is small if $e\left(R_{i}\right)=\omega$ for all $i$; otherwise we say $R$ is large.

Theorem 27. a) Let $R \cong \prod_{i=1}^{r} R_{i} \times A$ be a small Euclidean ring. Then

$$
e(R)=r \omega+\ell(A),
$$

where $n$ is the length of the Artinian principal ring A.

b) For every ordinal $\alpha<\omega^{2}$, there is a small Euclidean ring $R$ with $e(R)=\alpha$.

Proof. a) By Theorem 25, $e(A)=\ell(A)<\omega$. Now we apply the Product Theorem:

$$
r \omega+\ell(A)=e_{1}(R)+\ldots+e_{r}(R)+e(A) \leq e(R) \leq \bigoplus_{i=1}^{r} e\left(R_{i}\right) \oplus \ell(A)=r \omega+\ell(A) .
$$

b) The ordinals less than $\omega^{2}$ are of the form $r \omega+n$ for $r, n \in \omega$. By part a),

$$
e\left(\mathbb{C}[t]^{r} \times \mathbb{C}[t] /\left(t^{n}\right)\right)=r \omega+n .
$$


Are all Euclidean rings small? If so, Theorem 27 would be the ultimate result on Euclidean order types. This question was implicit in [Mo49] and made explicit in [Sa71. It was later answered negatively by Hiblot [Hi75], Hi77] and Nagata [Na78. It seems that the Euclidean order type of these large Euclidean domains has never been investigated...and, alas, will not investigated here. However, the following result shows that Euclidean rings of the sort familiar in number theory are small.

Proposition 28. (Sa71, Prop. 15]) Let $R$ be a Euclidean domain such that $R /(a)$ is a finite ring for all $a \in R^{\bullet}$. Then $R$ is small.

Proof. If not, there is $b \in R$ with $\varphi_{R}(b)=\omega$. Let $\bar{\varphi}: R /(b) \rightarrow$ Ord be the quotient Euclidean function. Write the elements of $R /(b)$ as $\overline{x_{1}}=0, \overline{x_{2}}, \ldots, \overline{x_{n}}$. For all $i>1$, $\bar{\varphi}\left(\overline{x_{i}}\right)<\bar{\varphi}(\overline{0}) \leq \omega$. But $\bar{\varphi}(\overline{0})=\sup _{i>1} \bar{\varphi}\left(\overline{x_{i}}\right)+1<\omega$. Thus, there exists $b^{\prime} \in(b)$ with $\varphi_{R}\left(b^{\prime}\right)<\omega$, contradicting the fact that $\varphi_{R}$ is isotone.

\section{Length Functions on Rings}

\subsection{The length function on a Noetherian ring.}

In this section we closely follow work of Gulliksen Gu73] and Brookfield Br02].

Let $R$ be a ring, and let $\mathcal{I}(R)$ be the lattice of ideals of $R$. Then $\mathcal{I}(R)$ is Noetherian (resp. Artinian) iff $R$ is Noetherian (resp. Artinian). Thus the dual lattice $\mathcal{I}^{\vee}(R)$ is Artinian (resp. Noetherian) iff $R$ is Noetherian (resp. Artinian).

Henceforth we suppose $R$ is Noetherian, so $\mathcal{I}^{\vee}(R)$ is Artinian with top element (0). By the results of $\S 1$ there is a least isotone map $\lambda_{R}: \mathcal{I}^{\vee}(R) \rightarrow$ Ord, the length function $\lambda_{R}$ of $R$, and we define the length of $\mathbf{R}$ as $\operatorname{len}(R)=\lambda_{R}((0))$.

For any ideal $I$ of $R, R / I$ is Noetherian, so $\lambda_{R / I}$ and $\operatorname{len}(R / I)$ are well-defined. If we denote the quotient map $R \rightarrow R / I$ by $q$, then the usual pullback of ideals $q^{*}$ identifies $\mathcal{I}(R / I)$ with an ordered subset of $\mathcal{I}(R)$ and hence also $\mathcal{I}^{\vee}(R / I)$ with an ordered subset of $\mathcal{I}^{\vee}(R)$, and it is easy to see that under this identification we have

$$
\left.\lambda_{R}\right|_{\mathcal{I}^{\vee}(R / I)}=\lambda_{R / I},
$$

and thus also

$$
\left.\operatorname{len}(R / I)=\lambda_{R / I}((0))\right)=\lambda_{R}\left(q^{*}((0))\right)=\lambda_{R}(I) .
$$

To ease notation, for $x \in R$ we put $\ell(x)=\lambda_{R}((x))$.

Proposition 29. Let $\varphi$ be a Euclidean function on $R$. For all $x \in R, \ell(x) \leq \varphi(x)$.

Proof. Since $R$ admits a Euclidean function, it is a principal ring, and thus $\mathcal{I}^{\vee}(R)=$ $R^{\bullet} / R^{\times}$. We may assume that $\varphi=\varphi_{R}$ is the bottom Euclidean function on $R$. Then both $\ell$ and $\varphi$ induce well-defined isotone functions on $R^{\bullet} / R^{\times}$. But by definition $\ell=\lambda_{R}$ is the least isotone function on $\mathcal{I}^{\vee}(R)$, so $\ell(x) \leq \varphi_{R}(x)$ for all $x \in R$.

If $R$ is a PID and $x \in R^{\bullet}$, then the ring $R /(x)$ is an Artinian ring and thus its length, which is equal to $\ell(x)$, is finite. In particular, for all $x \in R^{\bullet} \ell(x)<\omega$ and $\ell(0)=\omega$. From this the next result follows directly. 
Proposition 30. Let $R$ be a PID which is not a field, and let $x \in R$.

a) If $x \in R^{\times}$, then $\ell(x)=0$.

b) If $x \in R^{\bullet} \backslash R^{\times}$, we may write $x=\pi_{1} \cdots \pi_{n}$ for not necessarily distinct prime elements $\pi_{1}, \ldots, \pi_{n}$, and then $\ell(x)=n$.

c) We have $\ell(0)=\operatorname{len}(R)=\omega$.

\section{2. $\ell$-Euclidean rings.}

A ring $R$ is $\ell$-Euclidean if the function $x \in R \mapsto \ell(x) \in$ Ord $^{\bullet}$ is a Euclidean function on $R$. The point is that if $\ell$ is a Euclidean function on $R$, it is then the least Euclidean function on $R$, so that $e(R)=\operatorname{len}(R)$.

Example 3.1: a) The ring $\mathbb{Z}$ is not $\ell$-Euclidean.

b) For a field $k$, the ring $k[t]$ is $\ell$-Euclidean iff $k$ is algebraically closed.

Example 3.2: A norm on a nonzero ring $R$ is a function $|\cdot| R \rightarrow \mathbb{N}$ such that $|x|=0 \Longleftrightarrow x=0,|x|=1 \Longleftrightarrow x \in R^{\times}$and $|x y|=|x||y|$ for all $x, y \in R$. A ring admitting a norm is necessarily a domain: if $x, y \in R^{\bullet},|x y|=|x||y| \neq 0$, so $x y \in R^{\bullet}$. An $\ell$-Euclidean domain admits a Euclidean norm: $x \in R^{\bullet} \mapsto 2^{x}$.

Proposition 31. A localization of an $\ell$-Euclidean domain is $\ell$-Euclidean.

Proof. Left to the reader.

Proposition 31 furnishes further examples of $\ell$-Euclidean domains, namely any ring between $\bar{k}[t]$ and its fraction field. One wonders about further examples.

Question 32. Is there a classification of $\ell$-Euclidean domains?

\section{REFERENCES}

[Br02] G. Brookfield, The length of Noetherian modules. Comm. Algebra 30 (2002), 3177-3204.

[Fl71] C.R. Fletcher, Euclidean rings. J. London Math. Soc. 4 (1971), 79-82.

[Gu73] T.H. Gulliksen, A theory of length for Noetherian modules. J. Pure Appl. Algebra 3 (1973), 159-170.

[He06] G. Hessenberg, Grundbegriffe der Mengenlehre. Göttingen, 1906.

[Hi75] J.-J. Hiblot, Des anneaux euclidiens dont le plus petit algorithme n'est pas à valeurs finies. C. R. Acad. Sci. Paris Sér. A-B 281 (1975), no. 12, Ai, A411-A414.

[Hi77] J.-J. Hiblot, Correction à une note sur les anneaux euclidiens: "Des anneaux euclidiens dont le plus petit algorithme n'est pas à valeurs finies" (C. R. Acad. Sci. Paris Sér. A-B 281 (1975), no. 12, A411-A414). C. R. Acad. Sci. Paris S er. A-B 284 (1977), no. 15, A847-A849.

[Mo49] T. Motzkin, The Euclidean algorithm. Bull. Amer. Math. Soc. 55 (1949), 1142-1146.

[Na78] M. Nagata, On Euclid algorithm. C. P. Ramanujama tribute, pp. 175-186, Tata Inst. Fund. Res. Studies in Math., 8, Springer, Berlin-New York, 1978.

[Na85] M. Nagata, Some remarks on Euclid rings. J. Math. Kyoto Univ. 25 (1985), 421-422.

[Sa71] P. Samuel, About Euclidean rings. J. Algebra 19 (1971), 282-301.

[ZS] O. Zariski and P. Samuel, Commutative algebra, Volume I. Van Nostrand, Princeton, New Jersey, 1958.

Department of Mathematics, Boyd Graduate Studies Research Center, University of Georgia, Athens, GA 30602-7403, USA

E-mail address: pete@math.uga.edu 